\title{
A Volatility and Persistence-Based Core Inflation
}


A Volatility and Persistence-Based Core Inflation

Tito Nicias Teixeira da Silva Filho and

Francisco Marcos Rodrigues Figueiredo 


\title{
IMF Working Paper
}

African Department

\section{A Volatility and Persistence-Based Core Inflation ${ }^{1}$ \\ Prepared by Tito Nícias Teixeira da Silva Filho and Francisco Marcos Rodrigues Figueiredo}

Authorized for distribution by Ali Mansoor

January 2015

\section{This Working Paper should not be reported as representing the views of the IMF.} The views expressed in this Working Paper are those of the author(s) and do not necessarily represent those of the IMF or IMF policy. Working Papers describe research in progress by the author(s) and are published to elicit comments and to further debate.

\begin{abstract}
Intuitively core inflation is understood as a measure of inflation where noisy price movements are avoided. This is typically achieved by either excluding or downplaying the importance of the most volatile items. However, some of those items show high persistence, and one certainly does not want to disregard persistent price changes. The non-equivalence between volatility and (the lack of) persistence implies that when one excludes volatile items relevant information is likely to be discarded. Therefore, we propose a new type of core inflation measure, one that takes simultaneously into account both volatility and persistence. The evidence shows that such measures far outperform those based on either volatility or persistence. The latter have been advocated in the literature in recent years.
\end{abstract}

JEL Classification Numbers: C43, E31, E52

Keywords: Core inflation, inflation, persistence, volatility, triple weighted.

Author’s E-Mail Addresses: tdasilvafilho@imf.org, francisco-marcos.figueiredo@bcb.gov.br

\footnotetext{
${ }^{1}$ The authors would like to thank Fabio Araujo, Andre Minella, Rogelio Morales, Rodrigo Garcia-Verdu, Ahmat Jidoud, Juan Sole, Irineu de Carvalho, Ari Aisen, Michael Atingi Ego and Ali Mansoor for valuable comments and suggestions as well as other seminar participants at the Research Department of the Central Bank of Brazil and at the African and Western Hemisphere Departments at the IMF.
} 


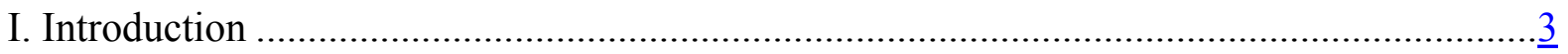

II. The Non-Equivalence Between Volatility and (the Lack of) Persistence...........................

III. Taking into Account both Volatility and Persistence .................................................... 1

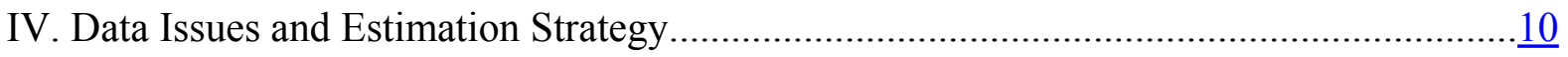

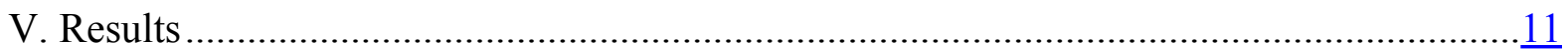

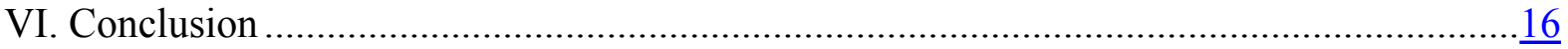

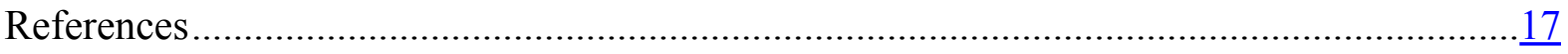

Figures

Figure 1. Brent Crude Oil Spot Prices (US\$ 1999 Prices)....................................................

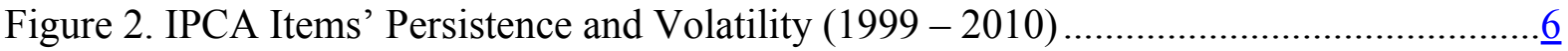

Tables

Table 1. Descriptive Statistics for IPCA Core Inflation Measures ......................................12

Table 2. Deviations from Trend IPCA Inflation .......................................................

Table 3. Forecasting Headline Inflation: Goodness of Fit and F-tests Results......................15 


\section{INTRODUCTION}

For most people core inflation is almost synonymous of an inflation measure that excludes volatile items. Indeed, core inflation is typically regarded as headline inflation less food and energy, two of the most volatile groups in the goods basket. For most people volatile groups just add noise to price indices.

Along time, many different measures of core inflation have arisen. However, most of them take the approach above as correct and differ simply in the precise way that volatile items are treated. This is the case, for example, of the trimmed mean core, where a given percentile from each tail of the individual price distribution is excluded. ${ }^{2}$ In this case, instead of excluding the same items in every period of time, as typical exclusion core measures do, items to be discarded are chosen following an objective statistical criterion (large price changes are excluded). Consequently, different items are excluded at different points in time.

Given the failure of those measures in some important dimensions [see, for example, Cecchetti (2006) and OECD (2005)], the so-called volatility measures - among others emerged. In such cases, volatile items are downplayed but not excluded.

One problem with the above approaches is that they all bear in common one flawed tenet: that when one assesses inflationary pressures volatile prices just bring noise to the outlook. However, volatility is not synonymous of noise.

Another crucial dimension in which prices changes should be analysed is persistence. If changes in the price of a given good are known to be quite persistent then those changes are likely to convey relevant information about inflationary pressures. More importantly, persistence also increases the chances of relevant second round effects. Hence, assuming that those changes are merely informationless noises, as the above approaches do, is a mistake that could entail important costs to policymakers.

Unfortunately, for both core inflation builders and policymakers evidence shows that volatility and persistence are not equivalent dimensions of price changes. In fact, many items considered too volatile, and hence usually discarded, are also highly persistent. An insightful and ironic example is precisely the price of oil, which is typically excluded by traditional core inflation measures.

Figure 1 shows the behaviour of the real price of oil from January 1999 to September 2012. As it can be seen, its relative price movements are usually long lasting. This is important since long lasting relative price changes are likely to produce relevant second round inflationary effects in the price chain. Moreover, excluding such items will create a

\footnotetext{
${ }^{2}$ Obviously, one can also take different percentiles from each tail of the distribution. This is precisely what the so-called asymmetric trimmed mean core does.
} 
permanent wedge between core and headline price indices. ${ }^{3}$ Therefore, based on the evidence below, one is not advised to exclude oil prices from core measures, no matter how volatile they are. ${ }^{4}$

Figure 1. Brent Crude Oil Spot Prices (US\$ 1999 Prices)*

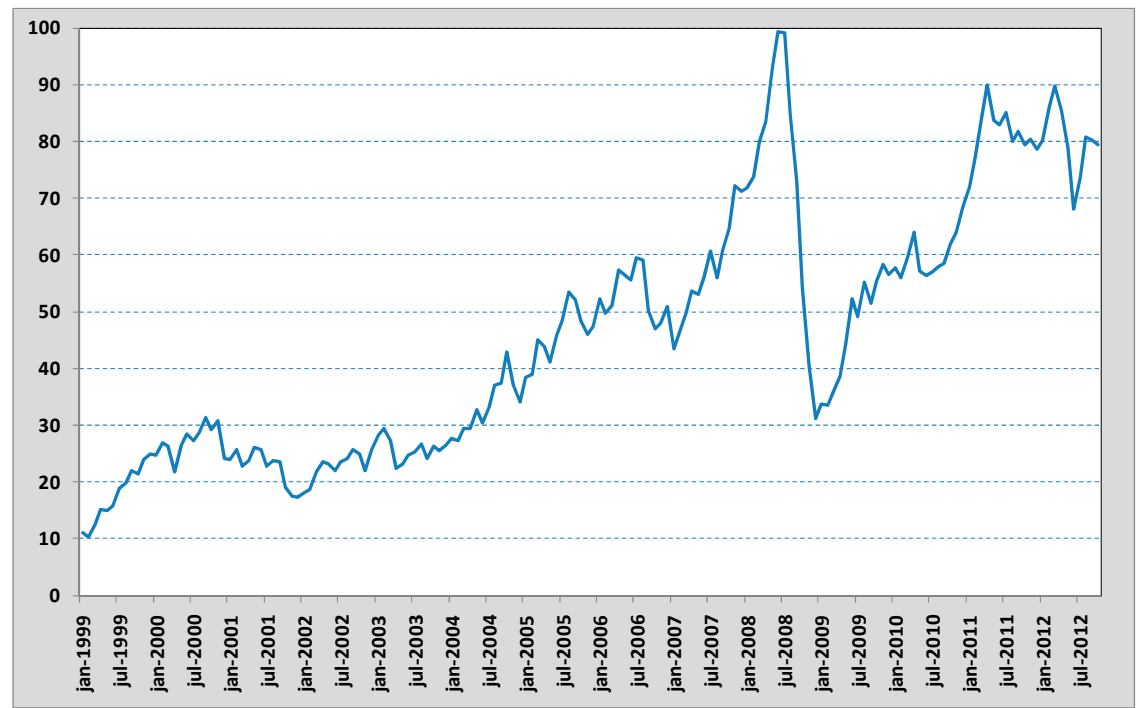

(*) Prices deflated by the U.S. CPI.

The steep and protracted increases in commodity prices in the last two decades are a testimony of the relevance and pervasiveness of such fact. Not surprisingly, Bryan and Cecchetti (1993) found evidence for the U.S. that headline inflation is a better predictor of itself than the traditional (ex-food \& energy) core. More disturbing evidence comes from Gavin and Mandal (2002), whose findings suggest that food prices do contain useful information about trend inflation in the U.S.

Despite the importance of the persistence dimension, only recently the core inflation literature has begun to pay attention to it. Indeed, Cutler (2001) seems to have been the first to have done so, inspired by the comments of Blinder (1997). A couple more papers are those of Demarco (2004), for Malta, Babestskii et al. (2007), for Czech Republic, Bilke and Stracca (2008), for the Euro area, and Rangasamy (2009), for South Africa. However, those papers took the "all or nothing approach". That is, they calculated core inflation using only the persistence dimension, while the volatility dimension was completely ignored.

\footnotetext{
${ }^{3}$ For some dreadful evidence on how the exclusion of items facing persistent price changes could produce a permanent and large wedge between core and headline inflation price indices see section 4 of da Silva Filho (2008).

${ }^{4}$ One can argue that oil prices should be excluded since they are "...for the most part, beyond the control of the central bank." (Blinder, 1997). However, this fact does not prevent pressure in those prices from disseminating through the economy. Moreover, using such rationale all commodity prices should be excluded, and not only oil prices.
} 
Thus, this paper proposes a new methodology for building core inflation measures: one that takes into account simultaneously both volatility and persistence. In such a case the original weights of each item is not only resized according to their volatility but are also further reweighted by their persistence. In that way, both volatility and persistence are taken explicitly into consideration. For example, if a given good is both highly persistent and volatile its reweighing could end up producing just minor differences compared to its original weight, in contrast to the situation in which just one criterion is used. However if a good is very volatile but little persistent or little volatile but highly persistent then its relative importance in the measure proposed above will be, respectively, much lower and higher in comparison to using the persistence or volatility criteria alone.

We test this new type of core inflation using Brazilian data. This should provide a very stringent test since not only Brazil is one of the leading emerging economies, having a welldiversified economy, but also has a history of frequent economic shocks, posing though challenges for a core inflation measure. The results show that this new type of core inflation measure has a good performance relatively to those currently used by the Brazilian Central Bank (BCB). Moreover, not only it outperforms volatility core measures but also those proposed by Cutler (2001), Demarco (2004), Babestskii et al. (2007), Bilke and Stracca (2008) and Rangasamy (2009), which are based either on persistence alone or on persistence and expenditure, ignoring the volatility dimension.

The paper is organised as follows: Section 2 shows evidence, using Brazilian data, that volatility and (the lack of) persistence are not redundant dimensions. Section 3 derives the entire family of volatility-persistence core inflation measures. Section 4 explains the data and the estimation strategy. Section 5 shows the results and assesses the performance of the new measures. Section 6 concludes the paper.

\section{The Non-Equivalence Between Volatility and (The Lack Of) Persistence}

The implicit assumption behind the removal of volatile items from core inflation measures is not only that those price changes are volatile but mainly temporary. If this is the case then the decision makes sense since, say, a 10\% increase in the price of lettuce due to bad weather conditions is likely to be completely reversed soon, once weather conditions are back to normal. ${ }^{5}$ However, this might not be the case, as Figure 1 has shown for oil prices.

Figure 2A displays further evidence on this regard by displaying the scatter plot between volatility and persistence for all 52 IPCA items. ${ }^{6,7}$ Two aspects stand out. First, there is one clear outlier: the item "Tubers, Roots and Legumes". Note that this item is the perfect

\footnotetext{
${ }^{5}$ More rigoursly, the relative price increase is expected to be completely reversed.

6 IPCA stands for Broad Consumer Price Index. It is the official inflation-targeting index in Brazil. Its components are defined, respectively, from the most to the least aggregation level as group, sub-group, item and sub-item.

${ }^{7}$ Section 3 and 4 explain how persistence and volatility are measured, respectively.
} 
example of what kind of item should be excluded from a good core measure, given that it is both extremely volatile and little persistent. ${ }^{8}$ Second, it is also obvious that volatility and persistence are only weakly correlated. ${ }^{9}$ Ideally, as argued, if one is willing to exclude the most volatile items in the price index that relation should be clearly negative. However, note that this might yet be the case when the sample is restricted to the most volatile items.

Therefore, Figure 2B shows the evidence when the sample is divided into two halves and only those items whose volatility is above the median are considered. As it can be seen, the evidence remains the same, as no significant correlation is found. Notice that the item "green vegetables" now stands out, since it is highly volatile and has almost zero persistence. This is another clear example of an item that should be excluded when building a core measure. ${ }^{10}$ Figure $2 \mathrm{C}$ goes one-step further and presents the evidence for only those items that are traditionally excluded (i.e. food and energy). The evidence remains unaltered ( $\mathrm{t}$-statistic equals to -1.13).

Figure 2. IPCA Items' Persistence and Volatility $(1999-\mathbf{2 0 1 0})^{11}$
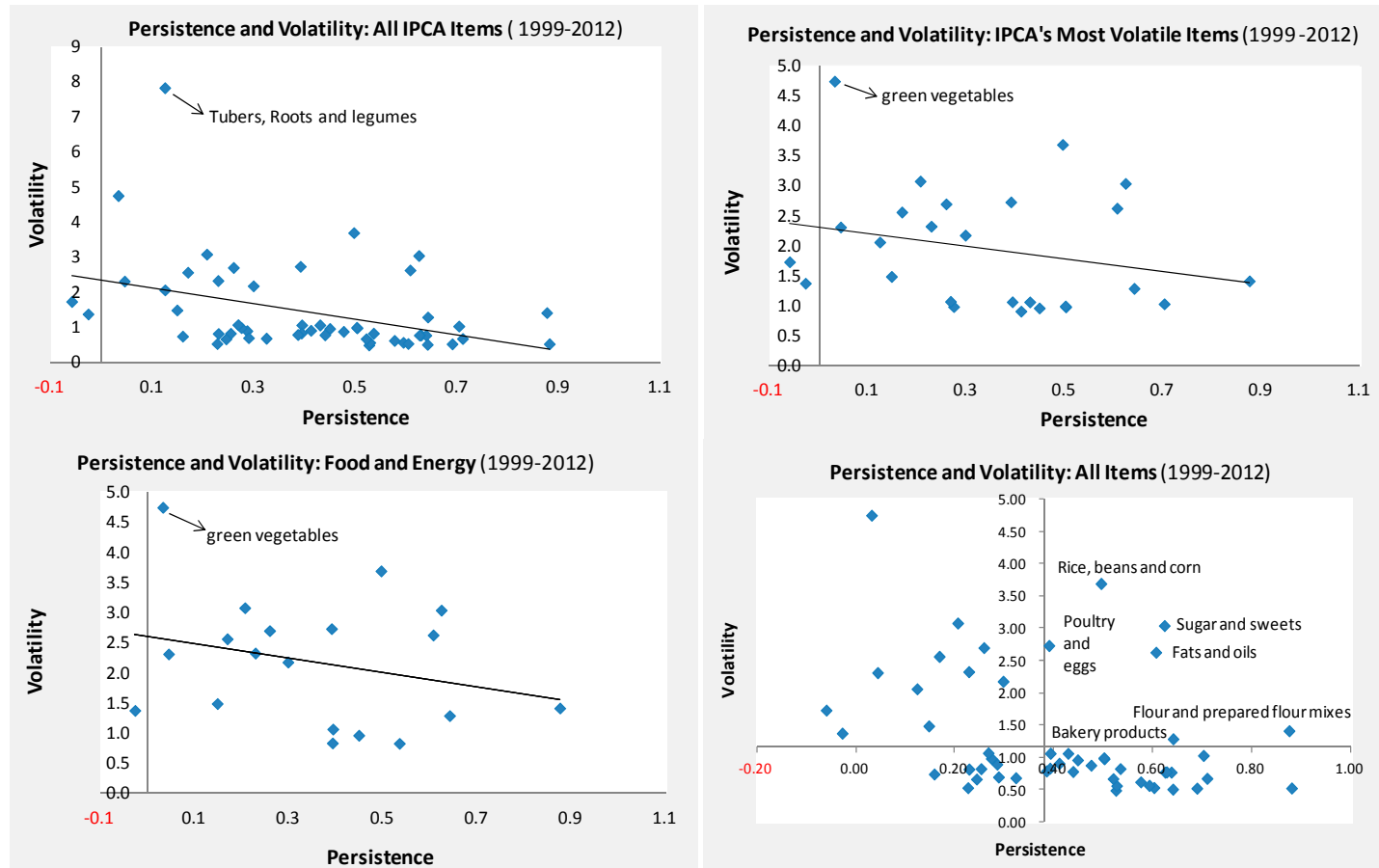

8 Given its outlier status it is excluded from panels B, C and D in order to improve inference and make the exposition clearer.

9 The correlation when the item "Tubers, Roots and Legumes" is excluded is -0.36 .

10 The regression of volatility on a constant and persistence produces a slope coefficient with a t-statistic equals to -0.96 .

11 Figure panels are lettered notionally as A, B, C and D, row by row. 
Finally, Figure 2D draws the axis according to the median volatility and persistence, so that each item is placed in its relative position (i.e. above or below the median) according to these two criteria. Note that the upper right quadrant shows those items that are both among the most volatile and the most persistent. Several items lie in that quadrant and therefore they are not good candidates for exclusion, since their exclusion is likely to imply loss of information. Nonetheless, they are usually excluded, as they are part of the food group. Therefore, in opposition to the common practice the evidence shows that they should not be discarded.

Given the evidence above it seems a sensible strategy to look at both volatility and persistence when choosing those items in the goods basket that should be excluded or reweighted.

\section{Taking into Account both Volatility And Persistence}

One key issue when considering persistence is obviously how to measure it. While the standard deviation is usually accepted to be a good measure of volatility, things are not that simple with persistence. Indeed, the literature lists several methods for estimating persistence, such as the largest autoregressive root (e.g. Cogley and Sargent, 2001), the number of times the mean of inflation is crossed during a given period (Marques, 2004) and the half-life (Pivetta and Reis, 2007). ${ }^{12}$ However, the most common method is the sum of the autoregressive coefficients in an univariate inflation equation. According to Andrews and Chen (1994), this method provides the best scalar measure of persistence.

Therefore, in this paper the sum of the coefficients of an $\operatorname{AR}(q)$ model is used as a measure of persistence. For each IPCA item $j$ we run the following recursive regression using monthly, seasonally adjusted, data:

$$
\pi_{j, t}=\alpha_{j}+\sum_{i=1}^{q_{j}} \rho_{j, t-i} \pi_{j, t-i}+\varepsilon_{j, t}
$$

where $\pi_{j, t} \equiv \ln P_{j, t}-\ln P_{j, t-1}$ is item's $j$ monthly inflation and $P_{j, t}$ is the monthly IPCA (Broad Consumer Price Index) price index for items $j=1,2, \cdots, 52$.

The degree of persistence is given by:

$$
\rho_{j, t}^{*}=\sum_{i=1}^{q_{j}} \rho_{j, t-i}
$$

where $q_{j}$ is the optimal lag length chosen according to the Schwarz criterion.

Once volatility and persistence are estimated one can calculate the "whole family" of volatility and persistence-based core inflation measures, which is done below.

The headline IPCA inflation is computed as follows

\footnotetext{
${ }^{12}$ It should be mentioned that while taking persistence formally into account is a new issue in the core inflation literature, there is a large literature concerned with the dynamic properties of inflation, mainly its persistence (see Fuhrer, 2010). Indeed, the efforts to better understand the persistence of inflation, its causes, as well as the effects of aggregation culminated in the creation of the Inflation Persistence Network (IPN) (see Altissimo et al., 2006).
} 


$$
\pi_{t}=\sum_{j=1}^{J} w_{j, t} \pi_{j, t}
$$

where $w_{j, t}$ is the original expenditure weight in time period $t$ for item $j$, subjected to the following restriction: $\sum_{j=1}^{J} w_{j, t}=1$.

A given core inflation measure $k$ can be generally described by: ${ }^{13}$

$$
\pi_{t}^{c(k)}=\sum_{j=1}^{J} \widetilde{w}_{j, t}^{k} \pi_{j, t}
$$

where $\pi_{t}^{c(k)}$ is the core inflation $k$ at period $t$, where $k$ indicates the methodology used to obtain the new set of normalized weights $\widetilde{w}_{j, t}^{k}$ such that $\sum_{j=1}^{J} \widetilde{w}_{j, t}^{k}=1$ for each $t$.

We begin describing the volatility-persistence family of core inflation measures by the two single-weighted measures that are based on either volatility $(v)$ or persistence $(p)$. In these cases the weights are given by ${ }^{14}$

$$
\begin{aligned}
& \widetilde{w}_{j, t}^{v}=\frac{\sum_{j=1}^{J} \sigma_{j, t}}{\sigma_{j, t}} \\
& \widetilde{w}_{j, t}^{p}=\frac{\rho_{j, t}^{*}}{\sum_{j=1}^{J} \rho_{j, t}^{*}}
\end{aligned}
$$

where $\sigma_{j, t}$ and $\rho_{j, t}^{*}$ are, respectively, the non-normalised relative volatility and the degree of persistence of item $j$ at time $t .^{15}$

One shortcoming of these measures is that they do not take into account the expenditure weights, which are used to compute the headline index. In order to avoid this potential problem, two other measures of the same family arise:

$$
\begin{aligned}
& \widetilde{w}_{j, t}^{d w(v e)}=\widetilde{w}_{j, t}^{v} w_{j, t} \\
& \widetilde{w}_{j, t}^{d w(p e)}=\widetilde{w}_{j, t}^{p} w_{j, t}
\end{aligned}
$$

Because they combine two sets of weights, they have been called in the literature double weighted measures of core inflation. The double weighted measure that combines volatility

\footnotetext{
13 This general expression fits most core inflation approaches, since they are essentially methods that reweigh the prices included in the headline index. To a summary of alternative ways to build core inflation measures, see Silver (2007) and Wynne (2008).

${ }^{14}$ The volatility-weighted index that is obtained using $\widetilde{w}_{j, t}^{v}$ is also known as "neo-Edgeworthian" index.

${ }^{15}$ The next section explains how volatility is calculated.
} 
and expenditure weights is indicated by the superscript $d w(v e)$, while the measure that uses persistence weights, instead of volatility, is indicated by the superscript $d w(p e){ }^{16}$

As discussed in the previous section, the non-equivalence between volatility and (lack of) persistence implies that the combination of the two dimensions is likely to provide valuable information about underlying inflation. Therefore, it is straightforward to think about another member of the family, where the final weights combine both persistence and volatility $d w(p v):^{17}$

$$
\widetilde{w}_{j, t}^{d w(p v)}=\widetilde{w}_{j, t}^{p} \widetilde{w}_{j, t}^{v}
$$

However, despite considering both dimensions those measures can also be criticized by not taking into account the original expenditure weights. Therefore, another member of the family naturally arises: the measure that combines the three sets of weights: expenditure, volatility and persistence (i.e. a triple weighted core measure). This measure is indicated by the superscript $t w$ (pve), and the weight is given by

$$
\widetilde{w}_{j, t}^{t w(p v e)}=\widetilde{w}_{j, t}^{p} \widetilde{w}_{j, t}^{v} w_{j, t}(10)
$$

Note that there is an alternative way of combining these three dimensions using each IPCA item's root mean square (one-step ahead) error (RMSE) from the $\operatorname{AR}(q)$ model given by (1). Since the conditional volatility comes from an autoregression, the degree of persistence is already taken into account. ${ }^{18}$ More precisely, in this case the core is given by

$$
\widetilde{w}_{j, t}^{t w(r m s e)}=\widetilde{w}_{j, t}^{r m s e} w_{j, t} \quad \text { where } \quad \widetilde{w}_{j, t}^{r m s e}=\frac{\sum_{j=1}^{J} r m s e_{j, t}}{r m s e_{j, t}}
$$

Also, note that, in practice, this is a double weighted measure, since it combines the set of weights obtained by the inverse of the root mean square error with the expenditure weights. However, given that the forecast error is conditional on the degree of persistence, this measure is a de facto triple weighted measure. Hence, this measure is indicated by the superscript $t w$ (rmse).

\footnotetext{
${ }^{16}$ Since March 2010, the Brazilian Central Bank has been releasing in its quarterly Inflation Report a double weighted core inflation measure based on volatility. The methodology of this core measure is described in da Silva Filho and Figueiredo (2011).

${ }^{17}$ As pointed out by an anonymous referee, the weighting scheme given by equation (9) is a particular case of $f\left(w_{j}^{v}, w_{j}^{p}\right)$ where more general solutions than that given by (9) can be devised such as $f\left(w_{j}^{v}, w_{j}^{p}\right)=$ $\left(w_{j}^{v}\right)^{a}\left(w_{j}^{p}\right)^{b}$ with $a, b>0$, as well as alternative ones such as $w_{j}^{v}+w_{j}^{p}$. However, the scheme in (9) displays a convenient property of being homogeneous of degree zero (the resulting weight is invariant to proportional changes in persistence and volatility) and, using our working sample, the results of the tracking inflation criterion obtained with weights given by (9) outperform those from the alternative scheme: $w_{j}^{v}+w_{j}^{p}$.

${ }^{18}$ We would like to thank Fabio Araujo, who has suggested this approach.
} 


\section{DATA ISSUES AND ESTIMATION STRATEGY}

The median debt-to-equity ratio has increased in the past 4 years, offsetting the deleveraging periods earlier in the decade (Figure 3). Overall, the median corporate had about the same debt-to-equity ratio in 2013 as it did in early 2003. The weaker tails of the distribution do not suggest a stronger deterioration of the debt-to-equity ratio amongst the most leveraged companies (figures 3 and 4).

In order to calculate the weights listed above the IPCA was disaggregated into its 52 items. ${ }^{19}$ The sample goes from January 1995 to December 2012. Note that it includes four changes in the structure of the expenditure weights, which followed the releases of more updated household expenditure surveys. Therefore, adjustments were made to make the data consistent along the sample. ${ }^{20}$

The relative volatility of each IPCA's item was calculated as follows:

$$
\sigma_{j, t}=\frac{1}{n-1} \sqrt{\sum_{i=1}^{n}\left(\hat{\pi}_{j, t-i}-\overline{\hat{\pi}}_{j, t}\right)^{2}}
$$

where: $\bar{\pi}_{j, t}=\frac{1}{n} \sum_{i=1}^{n} \hat{\pi}_{j, t-i}$ and $\hat{\pi}_{j, t}=\left(\pi_{j, t}-\pi_{t}\right)$.

The subscripts $j$ indexes each item in the goods basket, while $n$ gives the number of periods used in the calculation of the standard deviation of each item's relative inflation.

A 48-month moving window was used to calculate each item's volatility. This time-span seems to be a good compromise between a period long enough to produce reliable inferences and a period short enough to allow recent changes in volatility to be captured. Otherwise the series could be either too erratic or take very long to reflect changes in volatility. Note that since $j=48$ the working sample starts in January 1999 and the number of observations amounts to 168 .

As to the calculation of each item's inflation degree of persistence a recursive estimation was carried out instead, since the moving average strategy ended up producing estimates that were too volatile. It should also be noted that seasonally adjusted data was used, otherwise persistence estimates are likely to be biased downwards due to unmodelled seasonality.

The Schwarz information criterion was used to determine the autoregressive order $q_{j}$. In the search over $q_{j}$ twelve lags were used. However, given that results were unstable for certain items, the median lag length was chosen as the optimal lag. When $\rho_{j, i}^{*}$ was found to be

\footnotetext{
${ }^{19}$ See footnote 6 .

${ }^{20}$ A detailed description of the adjustments is available upon request.
} 
negative it has been set to zero, as in Cutler (2001) and Bilke and Stracca (2008). ${ }^{21}$ Finally, the weights were normalised to sum one at each period.

\section{Results}

This section presents evidence on the performance of the volatility-persistence family of core inflation measures, with particular interest in comparing those that take both volatility and persistence into account with those based only on persistence or on persistence \& expenditure. This comparison is important not only for the theoretical reasons laid out above but also because the latter have been increasingly advocated in the literature [see Cutler (2001), Demarco (2004), Babestskii et al (2007), Bilke and Stracca (2008) and Rangasamy (2009)]. It is also interesting to compare them with three core inflation measures currently calculated by the Central Bank of Brazil, named IPCA-EX, IPCA-DP and IPCA-MS.

The IPCA-EX is an exclusion-type core inflation measure, where the excluded items are defined based on both statistical and economic criteria. The IPCA-DP is a doubled weighted measure where each item's original expenditure weights are reweighed using the item's relative volatility, according to (7). ${ }^{22,23}$ The IPCA-MS is a smoothed trimmed mean core where the price of those items that changes infrequently during the year - mainly regulated \& administered prices changes - are "smoothed" before the trim. That is, the price changes are distributed along the month in which they occur and in the following eleven months. ${ }^{24}$

Table 1 displays some descriptive statistics for the measures cited above. With the exception of the persistence core note that all measures underestimated headline inflation to different degrees during the sample analysed (1999.1-2012.7). The annual bias ranges from -0.11 p.p. for the IPCA-DP to -0.46 p.p. for the DW-PV core. Most measures are much less volatile than headline IPCA, as expected. However, note that not only the two measures involving persistence that have been advocated in the literature (persistence and persistence \& expenditure) are very volatile but they are actually more volatile than headline inflation, a striking result. Obviously, those are undesirable features for a good measure of core inflation. Finally, none of the measures has a statistically significant bias. Even so, note that the magnitude of the IPCA-MS (0.37p.p.) and DW-PV (0.46p.p.) core measures are economically relevant.

\footnotetext{
${ }^{21}$ After seasonally adjusting the data only two items presented average (marginal) negative persistence $(-0.03$ and -0.06 for residential electricity and courses, respectively). This evidence suggests that findings of negative persistence are largely an outcome of misspecification.

${ }^{22}$ Note, therefore, that there is some overlap here, since the IPCA-DP is a member of the above family (equation 7).

${ }^{23}$ These two measures have been published since the March 2010 Inflation Report and their methodologies are fully described in da Silva Filho and Figueiredo (2011).

${ }^{24}$ This measure has been published since the Inflation Report of March 2001. For a description of the methodology, see Figueiredo (2001).
} 
Table 1. Descriptive Statistics for IPCA Core Inflation Measures

\begin{tabular}{l|ccccc}
\hline $\begin{array}{c}\text { Measures of Core } \\
\text { Inflation }\end{array}$ & $\begin{array}{c}\text { Monthly } \\
\text { Average }\end{array}$ & $\begin{array}{c}\text { Standard } \\
\text { deviation }\end{array}$ & $\begin{array}{c}\text { Coefficient } \\
\text { of Variation }\end{array}$ & $\begin{array}{c}\text { Annual Bias } \\
\text { (p.p.) }^{\mathbf{2}}\end{array}$ & $\begin{array}{c}\text { Unbiasedness } \\
\text { Test }^{\mathbf{3}}\end{array}$ \\
\hline Headline Inflation & $\mathbf{0 . 5 4}$ & $\mathbf{0 . 4 1}$ & $\mathbf{0 . 7 6}$ & - & - \\
\hline IPCA-EX & 0.52 & 0.28 & 0.54 & -0.24 & 0.62 \\
IPCA-DP & 0.53 & 0.29 & 0.54 & -0.11 & 0.82 \\
IPCA-MS & 0.51 & 0.20 & 0.40 & -0.37 & 0.39 \\
\hline SW-V & 0.52 & 0.36 & 0.70 & -0.19 & 0.71 \\
SW-P & 0.55 & 0.64 & 1.16 & 0.19 & 0.80 \\
DW-PE & 0.53 & 0.44 & 0.83 & -0.13 & 0.82 \\
DW-PV & 0.50 & 0.35 & 0.70 & -0.46 & 0.58 \\
TW-PVE & 0.52 & 0.27 & 0.50 & -0.17 & 0.71 \\
TW-RMSE & 0.52 & 0.25 & 0.44 & -0.20 & 0.66 \\
\hline
\end{tabular}

(1) W, DW and TW stand for single, double and triple weighted, respectively. V, P and E stand for volatility, persistence and expenditure, respectively. Combinations apply the same abbreviations. As described in the text IPCA-EX and IPCA-MS stand for exclusion core and smoothed trimmed mean core, respectively, while IPCA-DP is equivalent to DW-VE.

(2) This column gives the annualized value of the average monthly bias.

(3) P-values from unequal variances mean equality (unbiasedness) t-tests.

In order to check the ability of each core inflation measure to track the trend of headline inflation, the root mean squared error (RMSE) and the mean absolute deviation (MAD) were calculated (in relation to centred moving averages of 13, 25 and 37 months). Then the relative performance (i.e. in relation to headline inflation's RMSE or MAD) of each core was calculated.

Table 2 displays the results and, as can be seen, they are robust to the moving average period. Moreover, the qualitative results are similar to the evidence presented by Table 1 . The persistence-based measures that have been proposed in the literature show poor performance once again. The single-weighted persistence core advocated by Cutler (2001), Demarco (2004) and Bilke and Stracca (2008) presents by far the worst performance. In addition, the double-weighted persistence \& expenditure core advocated by Babestskii et al (2007) and Rangasamy (2009) bears the second worst performance. On the other hand, the two measures that take into account both volatility and persistence (alongside with expenditure weights) easily outperform the above two types of core measures - in accordance to our initial intuition - and are placed among the top 4 (in the RMSE criterion). ${ }^{25}$

\footnotetext{
${ }^{25}$ The good performance of the smoothed trimmed mean core in this criterion should be regarded with a grain of salt, since as shown in da Silva Filho and Figueiredo (2011) this measure has produced errors with poor dynamics (i.e. it sizably underpredicted as well as overpredicted inflation during long periods).
} 
Finally, a key evidence to assess how useful a given measure of core inflation is refers to its ability to predict headline inflation. More precisely, given the information set available to a forecaster, one would like to know if the additional information provided by the members of the volatility-persistence family helps to predict headline inflation. Given that that information set is a potentially very large one, this assessment will be done within a narrower scope.

Table 2. Deviations from Trend IPCA Inflation

\begin{tabular}{l|ccc|ccc}
\hline \multirow{2}{*}{$\begin{array}{c}\text { Measures of Core } \\
\text { Inflation }\end{array}$} & \multicolumn{3}{|c|}{ RMSE $^{2}$} & \multicolumn{3}{c}{ MAD $^{3}$} \\
\cline { 2 - 7 } & \multicolumn{2}{|c}{ Centered moving average } & \multicolumn{2}{c}{ Centered moving average } \\
\cline { 2 - 7 } & 13-month & 25-month & 37-month & 13-month & 25-month & 37-month \\
\hline \multirow{2}{*}{ IPCA-EX } & $0.60^{* * *}$ & $0.61^{* *}$ & $0.62^{* *}$ & $0.67^{* * *}$ & $0.68^{* * *}$ & $0.67^{* * *}$ \\
IPCA-DP & $0.67^{* * *}$ & $0.68^{* *}$ & $0.68^{* *}$ & $0.69^{* * *}$ & $0.70^{* * *}$ & $0.71^{* * *}$ \\
IPCA-MS & $0.48^{* * *}$ & $0.41^{* * *}$ & $0.40^{* *}$ & $0.50^{* * *}$ & $0.43^{* * *}$ & $0.43^{* * *}$ \\
\hline SW-V & 0.85 & 0.87 & 0.87 & $0.85^{*}$ & $0.84^{* *}$ & $0.85^{* *}$ \\
SW-P & $1.69^{* * *}$ & $1.63^{* * *}$ & $1.62^{* * *}$ & $1.74^{* * *}$ & $1.68^{* * *}$ & $1.64^{* * *}$ \\
DW-PE & $1.11^{* *}$ & $1.09^{*}$ & $1.10^{*}$ & $1.15^{* *}$ & $1.13^{* *}$ & $1.12^{* *}$ \\
DW-PV & 0.82 & 0.85 & 0.85 & $0.79^{* *}$ & $0.81^{* *}$ & $0.84^{* *}$ \\
TW-PVE & $0.67^{* *}$ & $0.66^{* *}$ & $0.67^{* *}$ & $0.71^{* * *}$ & $0.70^{* * *}$ & $0.71^{* * *}$ \\
TW-RMSE & $0.63^{* *}$ & $0.61^{* *}$ & $0.60^{* *}$ & $0.68^{* * *}$ & $0.65^{* * *}$ & $0.66^{* * *}$ \\
\hline
\end{tabular}

$*, * *$ and $* * *$ indicate significance at $10 \%, 5 \%$ and $1 \%$, respectively, in Diebold \& Mariano's test for predictive accuracy. The test aims at testing the null hypothesis of equality of expected forecast accuracy against the alternative of different forecasting ability across models, where forecast accuracy is measured according to a chosen loss function.

(1) SW, DW and TW stand for single, double and triple weighted, respectively. V, P and E stand for volatility, persistence and expenditure, respectively. Combinations apply the same abbreviations. As described in the text IPCA-EX and IPCA-MS stand for exclusion core and smoothed trimmed mean core, respectively, while IPCA-DP is equivalent to DW-VE.

(2) Root mean square error relative to that of headline inflation

(3) Mean absolute deviation relative to that of headline inflation

Among the myriad of variables that are part of the information set used by a forecaster, it seems obvious to argue that the history of inflation is certainly present. Therefore, the test used here aims at checking if - conditional on past headline inflation - core inflation brings any useful information to explain future headline inflation. If core inflation is uninformative when one controls for headline inflation it is very unlikely to be helpful once other variables are added to the forecasting model.

One way to make this assessment is to use a specification like (13), which produces multistep headline inflation forecasts. Three forecast horizons will be focused here: 2, 3 and 4 quarters. Those horizons are in accordance with estimates on the monetary policy 
transmission lag for Brazil. ${ }^{26}$ Moreover, good one-year ahead inflation forecasts (the longest horizon analysed) are a challenge not only in Brazil but in developed countries as well.

$$
\Delta_{k} p_{t+k}=\mu+\sum_{i=0}^{I} \alpha_{i} \Delta p_{t-i}+\sum_{j=0}^{J} \beta_{j} \Delta p_{t-j}^{c}+\varepsilon_{t+k}
$$

Where $\Delta_{k} p_{t} \equiv \ln P_{t}-\ln P_{t-k}$ is inflation over $k$ quarters, $P_{t}$ is the quarterly IPCA price index and $\Delta p_{t}$ and $\Delta p_{t}^{c}$ are, respectively, quarterly headline and core inflation. ${ }^{27}$ Note that equation (13) provides multi-step forecasts, and one consequence of such a setup is that forecasting errors will have moving average dynamics, since forecast periods overlap. This feature makes inference unreliable, and potentially wrong, since traditional standard errors statistics are invalid. One way to deal with this issue is to use corrected standard errors.

Table 3 provides evidence on the usefulness of the above core measures in predicting headline inflation over the near future in Brazil based on (13). The first line (in bold) shows the results for a restricted version of equation (13), one in which only lags of headline inflation enter the model (i.e. the betas are set to zero). The subsequent lines show the results when each core inflation measure is added to that restricted specification, one at a time.

Two results stand out: first, the extremely low $\mathrm{R}^{2}$ statistic, which shows that headline and core inflation over the previous four quarters are not very informative about inflation over the next 2, 3 and 4 quarters, during the sample analyzed, for Brazil. More strikingly, in almost all cases when core inflation is added to the model the equation's standard error actually increases, suggesting that core inflation measures are not very informative in explaining inflation developments up to one year ahead.

In this regard, note that only the model that includes the core measure that takes both volatility and persistence (along with expenditure) into account the equation's standard error decreases in all three horizons (shaded cells). This does not occur with either the singleweighted persistence core or the double-weighted persistence and expenditure core. This evidence concurs with our initial assessment about the need to consider both dimensions.

Second, the very limited role played by core inflation measures in explaining headline inflation over the near future in Brazil. Table 3 provides F-test results for the null that all the coefficients attached to lags of each core inflation measure are zero, and the results are revealing. In almost all cases, once one controls for headline inflation, core inflation measures do not add any useful information for predicting headline inflation.

There are three exceptions: the DW-PE core, which seems to add relevant information in the 2 and 3-quarter ahead horizons, and both the IPCA-MS and TW-PVE, which seem to be

\footnotetext{
${ }^{26}$ Estimates for Brazil suggest that changes in the interest rate begin to act upon the inflation rate in a statistically significant way somewhere between six and twelve months ahead. See, for example, Bogdanski et al. (2000).

${ }^{27}$ Regardless of the frequency of the data used in the models, the core inflation measures used in this paper are constructed using monthly data, which are then aggregated to lower frequency data.
} 
relevant in all three horizons. However, as just pointed out above, although the first two measures add relevant information they do not seem to be much useful, since forecasts become less accurate when they are included in the model. ${ }^{28}$ The single exception is the TWPVE measure, as when added to the model it produces forecasts that are (marginally) more accurate (in all three horizons). ${ }^{29}$

Table 3. Forecasting Headline Inflation: Goodness of Fit and F-tests Results ${ }^{1}$

\begin{tabular}{|c|c|c|c|c|c|c|c|c|c|}
\hline \multirow{2}{*}{ Explanatory Variables $^{2}$} & \multicolumn{3}{|c|}{ 2Q Ahead } & \multicolumn{3}{|c|}{ 3Q Ahead } & \multicolumn{3}{|c|}{ 4Q Ahead } \\
\hline & $\mathrm{R}^{2}$ & $\hat{\sigma}$ & F-test & $\mathrm{R}^{2}$ & $\hat{\sigma}$ & F-test & $\mathrm{R}^{2}$ & $\hat{\sigma}$ & F-test \\
\hline Headline Inflation Lags Only & 0.13 & 1.74 & - & 0.09 & 2.36 & - & 0.09 & 2.79 & - \\
\hline + IPCA-EX lags & 0.15 & 1.81 & 0.98 & 0.12 & 2.44 & 0.73 & 0.11 & 2.90 & 0.64 \\
\hline + IPCA-DP lags & 0.17 & 1.78 & 0.31 & 0.14 & 2.38 & 0.29 & 0.13 & 2.86 & 0.21 \\
\hline + IPCA-MS lags & 0.18 & 1.81 & 0.00 & 0.14 & 2.41 & 0.00 & 0.13 & 2.87 & 0.00 \\
\hline + SW-V lags & 0.20 & 1.75 & 0.55 & 0.16 & 2.38 & 0.42 & 0.16 & 2.81 & 0.28 \\
\hline + SW-P lags & 0.18 & 1.78 & 0.24 & 0.16 & 2.38 & 0.09 & 0.15 & 2.84 & 0.31 \\
\hline + DW-PE lags & 0.17 & 1.78 & 0.05 & 0.11 & 2.44 & 0.00 & 0.10 & 2.91 & $0.10^{3}$ \\
\hline + DW-PV lags & 0.23 & 1.72 & 0.20 & 0.15 & 2.40 & 0.20 & 0.12 & 2.89 & 0.16 \\
\hline + TW-PVE lags & 0.22 & 1.73 & 0.05 & 0.19 & 2.33 & 0.01 & 0.19 & 2.77 & 0.01 \\
\hline + TW-RMSE lags & 0.18 & 1.78 & 0.80 & 0.12 & 2.40 & 0.88 & 0.14 & 2.85 & 0.39 \\
\hline
\end{tabular}

(1) In order to highlight the low explanatory power of both headline and core inflation lags over future inflation, the $\mathrm{R}^{2}$ and $\sigma$ (the equation's standard error) statistics come from equation (13) without intervention variables, since the inclusion of dummies produces a sharp increase in those fit indicators and could induce one to think that headline and core inflation (over the last four quarters) explain a sizable part of future inflation. F-tests, however, are based on equation (13) augmented by intervention variables. The following impulse dummies were required for model congruence (apart from autocorrelation, which holds by construction): 2002.4 and 2003.1 in the 2-quarter ahead case, the latter plus 2003.2 in the 3-quarter ahead case, and the latter plus 2003.3 in the 4-quarter ahead case. Numbers in the F-test column are p-values from testing the hypothesis that all core inflation lags are not relevant for predicting headline inflation. Due to autocorrelation, F-tests use Andrews' (1991) heteroscedasticity and autocorrelation consistent standard errors (HACSE). Shaded areas indicate rejection of the null hypothesis at 5\% significance level or lower. The (quarterly) estimation samples begin in 2000.3, 2000.4 and 2001.1 in the 2, 3 and 4-quarter horizons cases, respectively, ending in 2012.2.

(2) SW, DW and TW stand for single, double and triple weighted, respectively. V, P and E stand for volatility, persistence and expenditure, respectively. Combinations apply the same abbreviations. As described in the text IPCA-EX and IPCA-MS stand for exclusion core and smoothed trimmed mean core, respectively, while IPCA-DP is equivalent to DW-VE.

(3) The cell is not shaded since the actual p-value is above $10 \%$, which does not appear here due to rounding.

\footnotetext{
${ }^{28}$ Moreover, as Tables 1 and 2 show the former has poor statistical properties. For example, it is more volatile than headline inflation.

${ }^{29}$ Another interesting piece of evidence would be to analyze the directional forecast accuracy of the core measures (see Da Silva Filho, 2012). However, such an analysis would require its own paper.
} 
It is important to say at this point that apart from autocorrelation - which was handled using Andrews' (1991) heteroscedasticity and autocorrelation consistent standard errors (HACSE) - all models passed in all diagnostic tests. ${ }^{30}$

\section{Conclusion}

The concept of core inflation is intimately linked to that of volatility. Indeed, most methodologies are simply different ways of either excluding or downplaying volatile items. Therefore, the rationale behind such methodologies is the same: that volatile items basically bring noise to the assessment of the inflation outlook. However, this conclusion is potentially flawed.

Another key - and usually forgotten - dimension over which price changes should be analysed is persistence. If changes in the price of a given good are known to be persistent then they are likely to convey relevant information about inflationary pressures, as they are more likely to get all the way through the price chain (i.e. second round effects). The relevance of persistence (in the core inflation literature) has recently begun to be acknowledged by some economists, who have been advocating core inflation measures based on persistence.

However, a major drawback of such proposal is that they take an "all or nothing" approach, since they go to the other extreme and ignore the volatility dimension. The evidence presented here shows that volatility and (the lack of) persistence are not equivalent dimensions, though. This means that if one focus on either volatility or persistence alone relevant information will be thrown away.

Therefore, a new type of core inflation measure is proposed: one that takes simultaneously into account both volatility and persistence (along with expenditure). Indeed, the evidence presented in the paper using Brazilian data gives support to the assessment above by showing that core inflation measures that are built using this new methodology seem to outperform those that focus on the persistence or volatility dimensions individually, which present a rather poor performance. Moreover, the measure proposed here also outperforms the doubleweighted persistence and expenditure core, which have been recently proposed in the literature.

The results have potentially far-reaching consequences not only for those building core inflation measures but also mainly for those using them to analyze the inflation outlook ahead.

\footnotetext{
${ }^{30}$ The following diagnostic tests were carried out in all estimations: Autocorrelation, ARCH, Normality, Hetero test, hetero-X test and RESET test. For details, see Doornik and Hendry (2009).
} 


\section{References}

Andrews, D. W. K., 1991, "Heteroskedasticity and Autocorrelation Consistent Covariance Matrix Estimation." Econometrica, Vol. 59, 1991.

Andrews, D. and H.-Y. Chen, 1994, "Approximately Median-Unbiased Estimation of Autoregressive Models”, Journal of Business \& Economic Statistics, Vol. 12, N².

Altissimo, F., M. Ehrmann and F. Smets, 2006, "Inflation Persistence and Price-Setting Behaviour in the Euro Area - A Summary of the IPN Evidence.”, European Central Bank Occasional Paper N ${ }^{0} 46$.

Babetskii, I., F. Coricelli and R. Horváth, 2007, "Measuring and Explaining Inflation Persistence: Disaggregate Evidence on the Czech Republic", Working Paper $\mathrm{N}^{0} 1$, Czech National Bank.

Bilke, Laurent and Livio Stracca, 2008, “A Persistence-Weighted Measure of Core Inflation in the Euro Area", ECB Working Paper N ${ }^{0} 905$.

Blinder, Alan S., 1997, "Commentary on Measuring Short-Run Inflation for Central Bankers", Federal Reserve Bank of St. Louis Review (May/June).

Bogdanski, Joel, Alexandre A. Tombini and Sergio Werlang, 2000, "Implementing inflation targeting in Brazil." Banco Central do Brasil Working Paper $\mathrm{N}^{\circ} 1$.

Bryan, Michael F. and Stephen M. Cecchetti, 1993, "The Consumer Price Index as a Measure of Infation", NBER Working Paper, N 4505.

Bullard, James, 2011, “The FED Should De-emphasize Core Inflation”, Southeast Missourian 5/25/2011.

Cecchetti, Stephen G., 2006, “Core Inflation is an Unreliable Guide”, Financial Times 9/12/2006.

Cogley, T. and Thomas J. Sargent, 2001, “Evolving Post-World War II US Inflation Dynamics". NBER Macroeconomics Annual 16.

Cutler, Joanne, 2001, "Core Inflation in the UK". External MPC Unit Discussion Paper $\mathrm{N}^{0} 3$. Bank of England.

Da Silva Filho, T. N. T., 2008, "Searching for the Natural Rate of Unemployment in a Large Relative Prive Shocks's Economy: the Brazilian Case". Central Bank of Brazil Working Paper $\mathrm{N}^{\mathrm{O}} 163$. , 2012, “Are Core Inflation Directional Forecasts Informative?”, Central Bank of Brazil Working Paper $N^{\mathrm{o}} 266$. 
Da Silva Filho, T. N. T. and F. M. R. Figueiredo, 2011, "Has Core Inflation Been Doing a Good Job in Brazil?” Revista Brasileira de Economia, Vol. 65, N 2.

Demarco, Alexander, 2004, “A New Measure of Core Inflation for Malta”, Central Bank of Malta Quarterly Review 2004:2.

Doornik, Jurgen A. and David F. Hendry, and (2009). Empirical Econometric Modelling PcGive 13, London: Timberlake Consultants Press.

Figueiredo, Francisco M. R., 2001, “Evaluating Core Inflation for Brazil”. Central Bank of Brazil Working Paper $\mathrm{N}^{\mathrm{O}} 14$.

Fuhrer, Jeffrey C., 2010, “Inflation Persistence", Handbook of Monetary Economics, Benjamin M. Friedman and Michael Woodford (eds).

Gavin, William and Rachel Mandal, 2002, "Predicting Inflation: Food for Thought," Federal Reserve Bank of St. Louis, Regional Economist, January.

Marques, Carlos R., 2004, “Inflation persistence: Facts or artefacts?”, ECB Working Paper $\mathrm{N}^{\mathrm{o}} 371$.

OECD, 2005, “Measuring and Assessing Underlying Inflation”. Economic Outlook $\mathrm{N}^{\mathrm{0}} 77$, May.

Pivetta, F. and R. Reis, 2007, "The Persistence of inflation in the United States", Journal of Economic Dynamics and Control, Vol. $31 \mathrm{~N}^{\circ} 4$.

Rangasamy, Logan, 2009, "Inflation Persistence and Core Inflation: The Case of South Africa”. South African Journal of Economics, Vol. 77:3.

Silver, Mick, 2007, “Core Inflation: Measurement and Statistical Issues in Choosing Among Alternative Measures”, IMF Staff Papers, Palgrave Macmillan Journals, vol. 54(1), pages 163-190, May.

Wynne, Mark A., 2008, “Core inflation: a review of some conceptual issues”, Review, Federal Reserve Bank of St. Louis, issue May. 